\title{
Mammuthus (Mammalia, Proboscidea) à la croisée de la Beringia, avec une digression sur les mammifères
}

Pascal TASSY

Muséum national d'Histoire naturelle, Département Histoire de la Terre, USM203-UMR7207 CNRS MNHN UPMC, Centre de Recherches sur la Paléobiodiversité et les paléoenvironnements, case postale 38, 57 rue Cuvier, F-75231 Paris cedex 05 (France) ptassy@mnhn.fr

MOTS CLÉS

Mammalia,

Proboscidea, mammouth laineux, mammouth des steppes,

Beringia,

Pléistocène,

Holocène,

équilibres ponctués, phylogénie moléculaire.

KEY WORDS

Mammalia,

Proboscidea, woolly mammoth, steppe mammoth, Beringia,

Pleistocene,

Holocene, punctuated equilibria, molecular phylogeny.
Tassy P. 2009. - Mammuthus (Mammalia, Proboscidea) à la croisée de la Beringia, avec une digression sur les mammifères. Geodiversitas 31 (4): 993-1005.

\section{RÉSUMÉ}

Les dernières analyses moléculaires de l'ADN ancien de mammouths laineux de Sibérie et d'Amérique du Nord confirment l'âge très ancien de la différenciation de Mammuthus primigenius, il y a 600 000-800 000 ans, et renouvellent l'étude des modalités de la "transition" Mammuthus trogontherii-Mammuthus primigenius. On discute aussi des différentes façons de pratiquer l'interdisciplinarité en biologie de l'évolution à propos du mammouth laineux et des relations de parenté des Mammalia.

\section{ABSTRACT}

Mammuthus (Mammalia, Proboscidea) at the crossroads in Beringia, with a digression on mammals.

The latest DNA analyses of woolly mammoths confirm the antiquity of the differentiation of the species Mammuthus primigenius, c. 600 000-800 000 years ago. They renew also the study of the "transition" between Mammuthus trogontherii and Mammuthus primigenius. The different ways of practicing multiple approaches in evolutionary biology are evoked, with the examples of the woolly mammoth and of the interrelationships of Mammalia. 
Bientôt ce serait possible: renaître mammouth. Ou tout au moins laineux. Gérard Manset

(Libération no. 8581, 6 décembre 2008, p. 29)

\section{INTRODUCTION}

Comme cet article est dédié à Louis de Bonis, je me permets de débuter par un souvenir personnel. En 1972 j'ai eu le bonheur de participer à la première mission paléontologique franco-grecque en Macédoine, dirigée par Louis de Bonis (alors maître-assistant à l'Université Paris 6) et Jean Melentis (alors directeur du laboratoire de paléontologie de l'Université de Thessalonique). Sur les traces de Camille Arambourg qui fouilla la région en 1915 avec les zouaves du $2 \mathrm{e}$ régiment de marche d'Afrique, nous faisions de la prospection dans les ravins tertiaires aux alentours de Vathylakkos, munis de cartes allemandes. Nous sommes tombés sur des placages quaternaires d'où émergeait une molaire d'allure franchement éléphantine. Une fois dégagée, la molaire enthousiasma Jean Melentis qui, justement ce jour là, nous avait rejoints sur le terrain : «Du mammouth... et pas du primigenius mais du trogontherii!». Certes, les grandes découvertes des missions menées par Louis de Bonis surgirent plutôt du Miocène supérieur; je n'ai pourtant pas trouvé de meilleure accroche pour enraciner ce bref article puisqu'il concerne les apports respectifs de la biologie moléculaire et de la paléontologie sur la connaissance de l'évolution du genre Mammuthus Brookes, 1828, un genre charismatique s'il en est, toujours source de débats.

\section{LE CADRE GÉOLOGIQUE TRADITIONNEL}

Le mammouth laineux, Mammuthus primigenius (Blumenbach, 1799) est le dernier des mammouths, à la fois sur les plan phylogénétique et chronologique. Il est d'usage de dire qu'il est le descendant de M. trogontherii (Pohlig, 1885). La transformation graduelle des molaires de mammouths est attestée dans les archives paléontologiques depuis au moins $4 \mathrm{Ma}$. Si l'on exclut les espèces africaines, au statut plus que discutable, et que l'on ne se réfère qu'aux formes eurasiatiques et nord-américaines, l'évolution des molaires depuis $2 \mathrm{Ma}$ (augmentation du nombre de lames et de la fréquence laminaire, amincissement de l'émail, augmentation de la hauteur de la couronne) a été historiquement interprétée tout aussi bien en termes d'évolution spécifique de type anagénétique en Eurasie avec deux dispersions en direction de l'Amérique du Nord, qu'en termes de radiation adaptative.

Dans le premier cas (Maglio 1973), le parallélisme dans l'évolution des molaires est limité aux deux lignées eurasiatique et nord-américaine après la première dispersion via l'isthme de Béring il y a 1,5 Ma environ. En Eurasie la succession des espèces de mammouths est vue au travers de sous-espèces décrites à partir du degré d'évolution dentaire (huit espèces et sous-espèces selon Guérin \& Patou-Mathis [1996: 148]).

Dans le second cas (Osborn 1942), la radiation adaptative des mammouths est celle d'une sousfamille (Mammontinae Osborn, 1921) qui inclut selon Osborn quatre genres ainsi nommés: Archidiskodon Pohlig, 1888, Metarchidiskodon Osborn, 1934 (monospécifique), Parelephas Osborn, 1924, Mammonteus Camper, 1788 (non valide; c'est-à-dire Mammuthus), subdivisé en 47 espèces et sous-espèces, où les évolutions parallèles intéressent chacun des trois genres principaux et où l'évolution dirigée est appelée aristogenèse (Osborn 1933a, b).

Ouvrons une courte parenthèse sur ce concept d'aristogenèse où les proboscidiens fossiles ont joué un rôle déterminant. Osborn (1933a: 160) écrit: "Absolutely inevitable and germinally predetermined evolution, distinguished as aristogenic or always tending toward improvement, takes place in widely separated geographic areas, at the same or different evolutionary rates. The term aristogenesis applies to this germinal creative potentiality. » Pour ce qui est des proboscidiens, chaque cuspide des molaires est, pour Osborn, un aristogène, significatif d'un niveau évolutif particulier. Dans la correspondance entre Marcellin Boule, alors directeur du laboratoire de paléontologie du Muséum national d'Histoire naturelle à Paris (MNHN), et Henry Fairfield Osborn, directeur de l'American Museum of Natural History de New York, on peut 
trouver un échange savoureux. Osborn sollicite des informations sur diverses molaires conservées au MNHN afin d'illustrer la gradation à l'intérieur du genre Archidiskodon (lettres d'Osborn à Boule du 11 décembre 1929 et du 25 janvier 1930 conservées à l'USM203-UMR7207 du MNHN). Dans sa réponse Boule en profite pour reprocher, au passage, "à la Paléontologie américaine», de "ne pas tenir compte suffisamment des variations individuelles et de multiplier sans raisons suffisantes les genres et les espèces" (lettre de Boule à Osborn du 1er mars 1930). Vicissitudes de l'information paléontologique: processus majeur de l'évolution pour l'un, simple variation individuelle pour l'autre.

La proposition de Maglio (1973: 14) selon laquelle le genre rassemble les espèces proches formant une unité phylogénétique a pour conséquence l'attribution au seul Mammuthus de toutes les espèces de mammouths; une proposition généralement suivie, avec toutefois des exceptions concernant Archidiskodon (voir Lister 1996). Les espèces primitives de Mammuthus, non seulement africaines mais aussi le mammouth méridional d'Eurasie, Mammuthus meridionalis (Nesti, 1825) sont souvent affiliées à Archidiskodon (dont l'espèce type est Elephas meridionalis Nesti, 1825). Dans ce cas, le genre Archidiskodon est tenu pour un genre ancestral de Mammuthus, avec une continuité évolutive entre A. meridionalis et $M$. trogontherii (Garutt \& Tikhonov 2001 ; Bajgusheva \& Titov 2001): au-delà d'une nomenclature non phylogénétique on en reste à un schéma anagénétique standard.

En Eurasie, la plus ancienne espèce de Mammuthus (Archidiskodon inclus) est Mammuthus rumanus (Stefanescu, 1924) du Villafranchien inférieur (MNQ16) (Markov \& Spassov 2003). La plus récente est $M$. primigenius dont les derniers individus, petits (hauteur au garrot estimée à $2 \mathrm{~m}$, voire moins) se sont éteints il y a 4000 ans environ dans l'île de Wrangel (Vartanyan et al. 1993). En Amérique du Nord, c'est un mammouth aux dents primitives qui est à l'origine de la diversification in situ des mammouths nord-américains (M. columbi (Falconer, 1857) puis M. exilis (Stock \& Furlong, 1928), forme naine de l'archipel Californien), avant que le mammouth laineux n'emprunte à son tour l'isthme de Béring il y a environ 150000 ans.
Quant à $M$. trogontherii il est classiquement reconnu en Europe centrale et occidentale à partir de MNQ22, soit 850000 ans, pour s'éteindre il y a un peu moins de 300000 ans après avoir été «le plus grand éléphant d'Europe» (Guérin \& PatouMathis [1996: 148])

\section{CHANGEMENT D'IMAGE: LES ÉQUILIBRES PONCTUÉS}

Parmi les travaux récents sur l'évolution des mammouths ceux de Lister \& Sher (2001) et Lister et al. (2005) ont renouvelé sensiblement la conception du mode d'évolution et de spéciation. En donnant un rôle prépondérant à la géographie et à la diachronie entre niveaux évolutifs comparables des molaires, Lister \& Sher (2001) ont conclu que la spéciation des mammouths s'apparentait plus aux équilibres ponctués qu'au gradualisme phylétique ou anagenèse. En dépit des apparences, une telle conception (Fig. 1) n'est pas totalement étrangère aux idées traditionnelles. On s'aperçoit néanmoins que c'est bien la prise en compte de la géographie qui permet de construire le schéma. Il apparaît que les populations sibériennes, à deux reprises, se différencient les premières pour supplanter tardivement les populations d'Europe occidentale. En réalité, les dernières découvertes concernant les premiers $M$. trogontherii enracinent plus précisément l'espèce en Chine vers 1,5 Ma (Wei et al. 2003; Lister et al. 2005).

Il est patent que la nomenclature habituelle appliquée à un tel schéma (Tassy 2004b) ne correspond pas aux parentés supposées. Les principes «hennigiens» de définition de l'espèce dans la dimension chronologique (Westoll 1956: 60; Hennig 1966: 56-61; voir également Bonde 2001) considèrent l'espèce comme l'élément du continuum évolutif situé entre deux spéciations ou entre une spéciation et une extinction; autrement dit l'espèce est inféodée à la notion de branchement. De ce point de vue les espèces qui survivent à une (ou plusieurs) spéciations n’ont pas de sens phylogénétique.

Il se trouve que les paléontologues ne sont pas tous intéressés par le concept chronologique de l'espèce, aussi paradoxal que cela puisse paraître; ni par le 


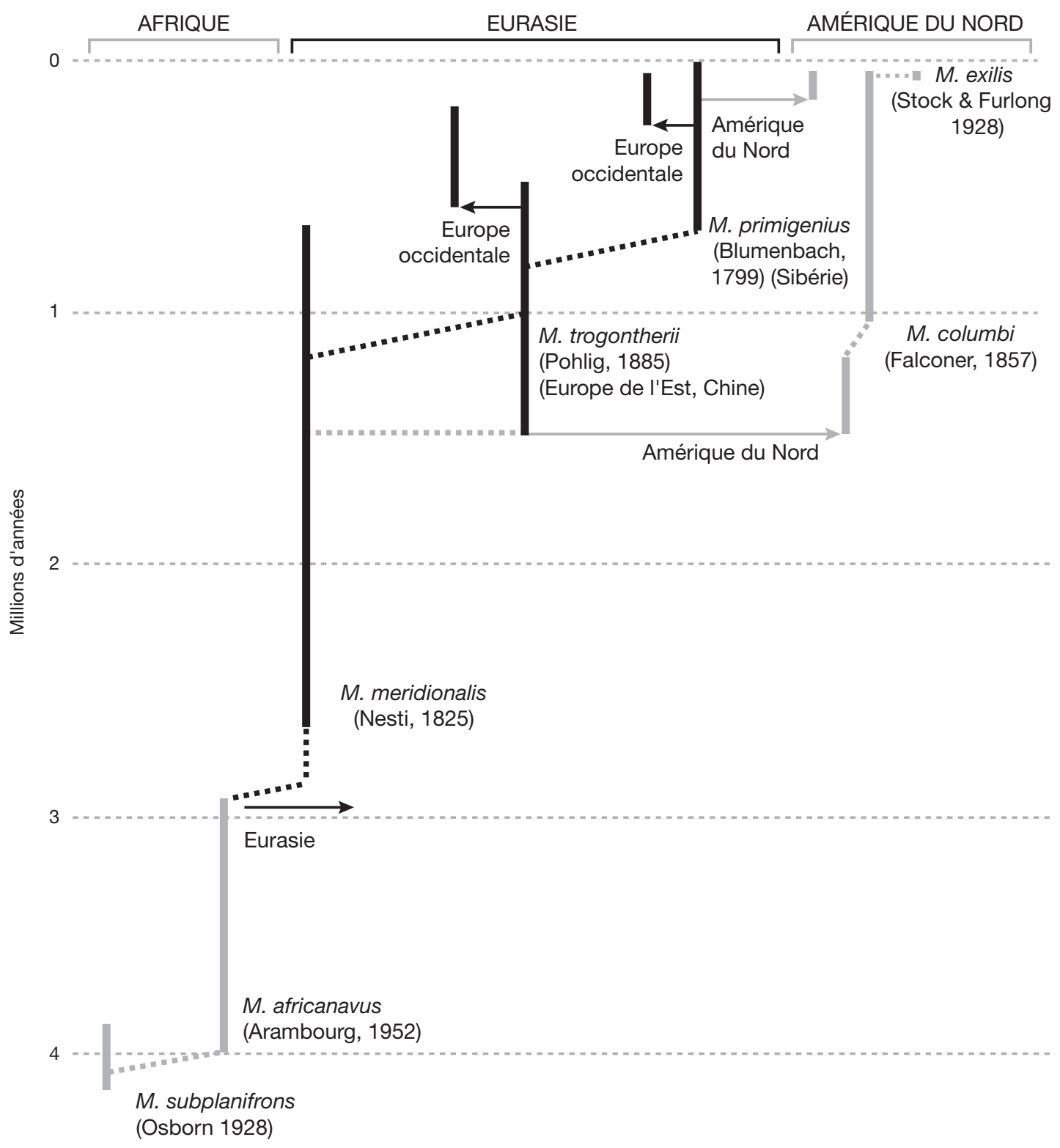

FIG. 1. - Évolution et spéciation chez le genre Mammuthus Brookes, 1828, correspondant au modèle des équilibres ponctués selon les travaux de Lister \& Sher (2001), modifié d'après Tassy (2004b). Selon Lister et al. (2005) l'extension chronologique de M. trogontherii (Pohlig, 1885) remonte à 1,5 Ma environ en Chine (voir texte).

concept phylogénétique, au demeurant, de telle sorte que la nomenclature traditionnelle n'a pas été bouleversée par ces résultats. Au reste, nous parvenons là aux limites de l'interprétation. Dans l'exemple original des équilibres ponctués (Eldredge
1971 ; Eldredge \& Gould 1972) le modèle de spéciation des équilibres ponctués considère les unités évolutives comme des populations, des sous-espèces géographiques. Les mêmes unités sont considérées ultérieurement par les mêmes auteurs comme des 
espèces (Eldredge 1986: 76; Gould \& Eldredge 1993: 223). L'usage dans le cas des mammouths de sous-espèces conçues comme des stades évolutifs (par exemple M. trogontherii wusti (Pavlow, 1910) M. trogontherii trogontherii (Pohlig, 1885) ne correspond pas nécessairement au concept géographique de la sous-espèce (ou espèce) tel que l'ont posé Lister \& Sher; d'autant que la différence entre "wusti» de Tiraspol en Moldavie (localité type) et «trogontherii» de Süssenborn (localité type) est loin d'être évidente (Dubrovo 1971; Lister 1996). Ainsi, sur la Figure 1 il n'est pas sûr que $M$. t. wusti de la base du Pléistocène moyen d'Europe centrale (Tiraspolien) (Schreve et al. 2007), aussi bien que "Archidiskodon aff. wusti» (au sens de Foronova 2001: 80) du bassin de Kuznetsk en Sibérie, puissent s'appliquer l'un et l'autre au segment qui précède la spéciation vers $M$. primigenius de Sibérie, ou bien au segment d'Europe de l'Est juste postérieur. Dans la même perspective, la résurrection du binom $M$. intermedius Jourdan, 1861 par Labe \& Guérin (2005) à la place de $M$. trogontherii chosaricus Dubrovo, 1966 ne résout pas vraiment le problème non plus, indépendamment des difficultés nomenclaturales récurrentes. Cette espèce est vue dans une conception anagénétique comme l'espèce intermédiaire entre $M$. trogontherii au sens strict et $M$. primigenius au sens strict (intermédiaire morphologique et chronologique (MNQ 24, 300 000-242000 ans) selon Labe \& Guérin (2005) depuis la Corrèze jusqu’à la Volga). Cependant, des molaires de cette époque, auparavant attribuées à Mammuthus primigenius s.s. (Lister 1996), comme à Steinheim et autres localités saaliennes sont attribuées désormais à $M$. trogontherii (Lister 2001; Lister \& Sher 2001; Lister et al. 2005). Mammuthus primigenius semble émerger de populations "trogontherii» il y a 700000 ans dans le nord-est de la Sibérie (Lister et al. 2005).

Il est un autre point soulevé par Lister \& Joysey (1992) et Lister \& Sher (2001: 1095). La fréquence laminaire, l'un des critères les plus (voire le plus) utilisés en biométrie des molaires d'éléphants, est ambiguë, voire trompeuse et, pour cela, abandonnée par ces auteurs (pour un même nombre de lames, une molaire plus petite aura une fréquence plus élevée sans que cela ait une signification évolutive). Peu les ont suivis, sans doute parce que les habi- tudes sont telles qu'il n'est pas possible de publier un article sur les éléphantidés - et donc les mammouths - sans y inclure ce paramètre: les reviewers rappelleraient vite à l'ordre les auteurs récalcitrants. Enfin, pour conclure cette section, mentionnons également l'aspect quantitatif de ce type d'enquêtes paléontologique et biostratigraphique. Les molaires forment la majeure partie du matériel fossile jalonnant les sites archéologiques du dernier million d'années lorsqu'émerge l'espèce $M$. primigenius. Il ne semble pas que l'on soit prêt à minorer cette source d'informations statistiques.

\section{LES PHYLOGÉNIES MOLÉCULAIRES}

Le mammouth laineux, et plus généralement les mammifères trouvés à l'état congelé, ne sont pas des fossiles comme les autres. Ils fournissent de l'ADN permettant des analyses phylogénétiques qui viennent en contrepoint des approches morphoanatomiques. Il y a peu je doutais de l'obtention rapide de l'intégralité de l'ADN mitochondrial à partir de différents tissus (Tassy 2004a). C'est désormais chose faite (Krause et al. 2006; Rogaev et al. 2006; Gilbert et al. 2008) et la course va même vers un génome complet (Poinar et al. 2006; Miller et al. 2008). J'ironisais par ailleurs sur la possibilité d'une résurrection du mammouth par clonage. Sur ce dernier point, on est sûr que ce ne sera pas pour demain, ni même pour après-demain (mais c'est pour 2059 nous assure Nicholls [2008], pince-sans-rire).

Il est loin le temps - une douzaine d'années seulement, toutefois - où obtenir un fragment infime d'ADN à partir d'os ou de poils de spécimens conservés en collection apparaissait comme un exploit. Cela dit, la controverse existe toujours sur la qualité de l'ADN que l'on peut respectivement séquencer à partir de l'os et des poils ainsi que sur les protocoles: on lira avec profit des commentaires aussi contrastés que ceux - triomphalistes - de Gilbert et al. (2007) et ceux - très critiques - de Debruyne et al. (2008b).

Après quelques travaux pionniers où les contaminations ont joué un rôle - par exemple le mammouth de Lyakhov (Îles de la Nouvelle-Sibérie) : le 
paradoxe de l'analyse de Yang et al. (1996) résolu par Debruyne et al. (2003) - les résultats récents obtenus pratiquement en routine soulèvent deux problèmes : la parenté du mammouth relativement aux éléphants d'Afrique et d'Asie, et les modalités de l'évolution de Mammuthus primigenius dans le temps et dans l'espace.

Premier problème: la recherche du groupe frère de Mammuthus. Celle-ci résume bien le conflit ou plutôt l'apparence de conflit - entre morphologie et molécules. Les paléontologues ont de tout temps reconnu une proche parenté entre Elephas et Mammuthus, en raison notamment de la similitude dans l'élévation de la région fronto-pariétale, que l'on pouvait déjà déceler sur la toute première illustration d'un crâne de $M$. primigenius de Sibérie due à Messerschmidt et publiée par Breyne (1741). Mais cette similitude intéresse des formes relativement tardives: on ignore encore aujourd'hui la morphologie crânienne des plus anciens mammouths africains aux alentours de $4 \mathrm{Ma}$ aussi bien que celle d'Elephas nawatensis Tassy, 2003; et celle d'E. ekorensis Maglio, 1970 est toujours bien imparfaitement connue (Maglio 1973: 34).

Initialement quelques données moléculaires (Noro et al. 1998; Thomas et al. 2000 ; Debruyne et al. 2003) ont indiqué une parenté (Mammuthus, Loxodonta), d'autres (Ozawa et al. 1997; Greenwood et al. 1999) une parenté (Mammuthus, Elephas), d'autres encore ne résolvaient pas le problème (Derenko et al. 1997; Barriel et al. 1999).

Les derniers bilans suggèrent avec circonspection, tant les résultats sont ténus (Capelli et al. 2006), une relation (Mammuthus (Loxodonta, Elephas) à partir de l'ADN nucléaire, ou bien, plus fermement (Krause et al. 2006; Rogaev et al. 2006; Gilbert et al. 2008), la relation (Mammuthus, Elephas) à partir du génome mitochondrial. Cependant, Krause et ses collaborateurs détaillent la précarité du résultat: extrêmement peu de divergence entre les trois genres, et des résultats contradictoires selon les algorithmes et les modèles de substitution nucléotidique (Krause et al. 2006: supplementary information). Nous n'avons donc là que de fortes présomptions d'une parenté (Mammuthus, Elephas). En revanche, ce qui est acquis est que les résultats moléculaires suggèrent une radiation rapide - potentiellement moins d'un million d'années - des trois genres éléphantins dès le Miocène terminal, une conclusion qui va dans les sens des découvertes paléontologiques liées aux Loxodonta et Elephas primitifs (Tassy 2003; Shoshani \& Tassy 2005). En conclusion on peut souligner la communauté d'approche vis-à-vis de cette question entre paléontologues et molécularistes.

Second problème: l'évolution moléculaire du mammouth. Celle-ci est source de surprises. Les travaux de Barnes et al. (2007), effectués à partir de l'analyse de 41 individus, ont mis en évidence une variation intraspécifique de $M$. primigenius interprétable en termes d'histoire généalogique de deux clades mitochondriaux attestés (un troisième étant suggéré). Un clade américano-sibérien se diversifierait en Sibérie il y a un peu plus de 60000 ans et un clade sibérien et occidental s'éteindrait il y a un peu plus de 40000 ans.

Ce modèle a été confirmé et extrêmement enrichi par Debruyne et al. (2008a). Ces derniers ont analysé 160 mammouths se distribuant en trois clades mitochondriaux (nommés respectivement A, B et C-D-E). La phylogéographie, obtenue à partir d'un échantillon pour la première fois équilibré entre mammouths de Sibérie et d'Amérique du Nord, montre une trifurcation basale avec un groupe $\mathrm{A}$ exclusivement asiatique, un groupe $\mathrm{B}$ américano-eurasiatique (deux spécimens seulement) et un groupe $\mathrm{C}$ exclusivement américain. Ce dernier est le groupe souche d'où émergent la plupart des mammouths eurasiatiques (groupes $\mathrm{D}$ et $\mathrm{E}$ ). Le sens de l'évolution géographique et de la dispersion populationnelle va de la Beringia Est vers la Beringia Ouest et l'Europe, d'où l'expression "out of America» forgée par Debruyne et al. (2008a). La chronologie postulée est surprenante. Entre 44000 et 5000 ans tous les mammouths sont des descendants du groupe souche américain C. Ce groupe se serait mis en place en Amérique du Nord il y a environ 400 000-500 000 ans (soit assez tôt dans le Wisconsinien américain, bien avant les 150000 ans habituellement avancés) avec un retour de populations de mammouths américains en Eurasie aux alentours de 300000 ans. Même si l'hypothèse n'a jamais été dominante, la possibilité d'échanges multiples via la Beringia a déjà été envisagée (Dudley 1996: 290) comme explication 
concurrente à celle, peut-être trop simple, d'une ancienne dispersion en Amérique d'un mammouth de grade évolutif $M$. meridonalis et celle récente d'un $M$. primigenius évolué. De la sorte, un réexamen taxinomique des mammouths nord-américains est à l'ordre du jour.

Quant aux premiers haplotypes de mammouths laineux exclusivement asiatiques (groupe A de Debruyne et al. [2008a]), ils remonteraient selon les estimations moléculaires à 800 000-600 000 ans, ce qui évoque la transition $M$. trogontherii- $M$. primigenius en Europe de l'Est et en Sibérie, voire l'espèce $M$. trogontherii elle-même. L'espèce morphologique $M$. trogontherii pourrait donc appartenir à l'espèce biologique $M$. primigenius. Relativement à $M$. primigenius, $M$. trogontherii est un mammouth de grande taille mais on sait que la taille n'est pas un trait irréversible. Ses molaires sont plus grandes et primitives mais peut-être faudrait-il revoir l'effet de taille: plus les molaires sont petites (celles de $M$. primigenius le sont), plus la fréquence laminaire est élevée et cela indépendamment de tout niveau évolutif. La paléontologie et la biologie moléculaire rencontrent donc ici un problème de spéciation (ou de définition de l'espèce) universel et $M$. trogontherii-le mammouth dit des steppes, comme si le mammouth laineux ne l'était pas également - est désormais prêt à recevoir les feux des projecteurs les plus médiatiques, ceux des télévisions et d'internet (Rincon 2008).

«Pas mal d'eau s'est écoulée dans le Jourdain depuis qu'on a abordé cette discussion" avait coutume de répéter mon regretté ami Hezy Shoshani (1943-2008). Lorsque Churcher (1980) s'est posé la question de savoir si les mammouths nordaméricains migraient, c'était dans le contexte de migrations saisonnières à l'échelle du continent américaine et de leur effet sur le comportement éventuellement prédateur d'Homo sapiens (même si le couloir représenté par la Beringia était pris en compte). Depuis, une telle interrogation a pris une toute autre dimension, lesdites "migrations" sont en réalité des dispersions de grande amplitude avec remplacements de populations américanoeurasiatiques.

On le voit: les interpénétrations entre les intérêts des molécularistes, des paléontologues, des strati- graphes et des paléoécologistes du Pléistocène et de la transition Pléistocène-Holocène sont nombreuses. Les datations des spécimens de mammouths sur lesquels travaillent molécularistes et paléontologues sont cruciales pour tous, que l'on s'intéresse à l'étude de la diversification des faunes quaternaires ou à celles des extinctions de leurs composants (Bocherens 2003; Guthrie 2004, 2006; Kuzmin \& Orlova 2004; Stuart et al. 2004), au point que l'on peut imaginer de beaux jours pour l'interdisciplinarité.

\section{LES MAMMALIA POUR FINIR}

L'exemple du mammouth laineux montre comment les approches moléculaire et morpho-anatomique se répondent, se complètent, se confrontent et ouvrent, ensemble, des perspectives communes de recherche, touchant notamment au passage $M$. trogontherii/M. primigenius et à la dénomination même de ces deux taxons. Il s'agit là d'un moment privilégié dans la recherche phylogénétique. Les spécialistes de mammouths ignorent les cloisons disciplinaires.

Ce qui est vrai au niveau spécifique devrait l'être à l'échelle de la classe des Mammalia. À ce sujet, la confrontation des résultats morpho-anatomiques et moléculaires est déjà une vieille histoire. Cependant le dialogue entre les deux communautés que forment les paléontologues et les biologistes des molécules ne va pas toujours de soi. Peu à peu, l'inexorable ascension des approches moléculaires liées à des modélisations probabilistes a marginalisé l'apport des recherches morphologiques. Ces dernières n'émergent pratiquement plus qu'au travers de recherches paléontologiques, à partir d'analyses de parcimonie engendrées, le plus souvent, par la découverte d'un nouveau fossile majeur. En outre, les travaux paléontologiques intéressent souvent des groupes de placentaires, de marsupiaux, ou de thériens sans aborder le cadre des Mammalia in extenso. Force est de constater qu'actuellement persistent bien des contradictions entre les résultats moléculaires et les résultats morpho-anatomiques. À lire dans l'ouvrage dirigé par Rose \& Archibald (2005) les articles écrits par les paléontologues et celui rédigé par les molécularistes, on peut presque 
se demander s'il s'agit bien des mêmes mammifères! Il y a là un double problème. Non seulement les sources d'information ne sont pas les mêmes mais, en outre, les méthodes analytiques sont différentes. Les modélisations bayésiennes des données moléculaires peuvent-elles être intrinsèquement comparées aux analyses de parcimonie des caractères morphologiques? Comment interpréter des groupements morphologiques induits par des fossiles avec des groupements moléculaires contradictoires? Cette question n'est pas nouvelle mais elle est en train de prendre un tour particulièrement aigu.

Pour un paléontologue cladiste un ordre de placentaire intégrant les données fossiles est défini par son morphotype ancestral, principe de base de la cladistique. L'ordre des Proboscidea, par exemple, a peu à voir avec la famille des Elephantidae si l'on pense à la morphologie crânienne et dentaire de Phosphatherium escuilliei de l'Éocène basal du Maroc (Gheerbrant et al. 2005). À l'inverse, dans la nature actuelle Proboscidea = Elephantidae, en tout cas sur le plan des caractères moléculaires à comparer avec ceux des autres mammifères. Raisonner en morpho-anatomie comme s'il s'agissait de molécules est certainement source d'erreurs. On peut, de ce point de vue, citer le cas du travail de Cox (2006) qui a cherché à retrouver des caractères morphologiques sur le crâne de représentants actuels de taxons de rang supérieur : le caractère "perte du foramen lacrymal» est signalé chez les Proboscidea et les Sirenia (Cox 2006: 518) alors que le foramen est présent chez les proboscidiens paléogènes et les siréniens paléogènes (Tassy 1981: 116; Savage et al. 1994: 431 ; Gheerbrant et al. 2005: 247). Pour en rester aux Proboscidea et autres paenongulés, des travaux morpho-anatomiques tendent à soutenir le groupement issu de la biologie moléculaire des Afrotheria (Tabuce et al. 2007) ou une partie des afrothères (Wible et al. 2007) mais le débat existe. Il existe même pour pratiquement tous les regroupements profonds de la classe des Mammalia.

En matière de phylogénie des Mammalia, la morpho-anatomie est en passe d'être numériquement supplantée par les molécules, si elle ne l'est pas déjà. Les phylogénies moléculaires des mammifères s'accumulent depuis des années. L'assurance des molécularistes est d'ailleurs telle que Waddell et al. (1999b) allèrent jusqu'à proposer pour la couverture d'un numéro de Systematic Biology, un numéro sur l'origine des ordres de mammifères, un arbre moléculaire des Mammalia révolutionnaire, avec dénomination de taxons nouveaux pour les dichotomies profondes. Cet arbre n'avait toutefois pas été obtenu par un quelconque gène (et encore moins par concaténation) mais simplement par compilation «à la main » de certaines parties de divers arbres. On est loin ici du sacro-saint (et naïf?) binôme contrôle/ réfutation de la cladistique. Dans cet arbre où l'on découvre les groupes Atlantogenata et Laurasiatheria comme issus de la dichotomie de base des placentaires, figurent entre autres les Zooamata (situés à $70 \mathrm{Ma}$ ) avec les périssodactyles groupe frère des carnivores et des pholidotes. Je m'étais alors amusé à voir quels étaient les groupements moléculaires communs dans les différents articles formant le corps du numéro. Pour prendre l'exemple des périssodactyles - aux parentés relativement stables - on les trouve néanmoins indéterminés dans un clade comprenant également les pholidotes, carnivores et cétartiodactyles (Gatesy et al. 1999: 17), ou groupe frère des carnivores (Waddell et al. 1999a: 35; Penny et al. 1999: 83) ou de l'ensemble carnivorescétartiodactyles (Waddell et al. 1999a: 40), ou bien indéterminé dans les placentaires (Liu \& Miyamoto 1999: 58) ou groupe frère des chiroptères (Liu \& Miyamoto 1999: 59), ou encore groupe frère des cétartiodactyles (représentés par la vache) aussi bien que de l'ensemble vache-hérisson (Springer et al. 1999: 69). Autrement dit la parenté étroite périssodactyle-carnivores revient le plus souvent (deux fois) mais ce n'est pas cette parenté qui est retenue dans les Zooamata par Waddell et al. (1999b).

On me dira que des travaux moléculaires de dix ans d'âge n'ont plus besoin d'être commentés. De fait, depuis lors, les résultats moléculaires se sont accumulés pour être tout dernièrement synthétisés par Fabre (2008: 45) où est retenu le taxon Pegasoferae Nishihara, Hasegawa \& Okada, 2006, dont la structure de parenté est (Chiroptera (Perissodactyla (Pholidota, Carnivora))) ; cependant deux publications moléculaires (Matthee et al. 2007 et Springer et al. 2007) n'ont pas retrouvé ce taxon qui passe pourtant pour être fondé sur des insertions rares dans les rétroposons L1 (Nishihara et al. 2006). 
L'obtention d'un consensus entre données moléculaires n'est donc pas dénué de difficultés; celle d'un consensus avec les données morpho-anatomiques est par conséquent d'autant plus difficile à atteindre. Au point que Springer et al. (2007) ont tout simplement proposé d'abandonner une bonne fois pour toutes les données morphologiques puisqu'elles s'avèrent incapables de retrouver les clades de placentaires découverts par les molécules. La confrontation des données n'est donc plus à l'ordre du jour et, par voie de conséquence, si un tel point de vue devait orienter les recherches phylogénétiques futures, anatomistes et paléontologues n'en seraient plus. Ce n'est pas une mince suggestion.

Des deux sources d'information phylogénétique l'une d'entre elle, la morpho-anatomie, serait-elle de trop? Dans une réponse élaborée, Asher et al. (2008) ont, entre autres arguments, essayé de faire comprendre que sans données morpho-anatomiques il n'est pas de paléontologie, et sans paléontologie pas de possibilité de contrôle des propositions fondées sur les seules formes vivantes. La réponse de Springer et al. (2008) ne laisse de surprendre. Je ne retiens ici qu'un aspect de leur argumentation. La question du contrôle mutuel des résultats moléculaires et paléontologiques ne représente que $14 \%$ de leur article; mais c'est surtout pour dire que les morphologistes doivent être soucieux de leurs propres difficultés et que "The problem of teasing homplastic ( $\mathrm{sic}$ ) morphological markers from homologous morphological markers will not simply disappear if ignored» (Springer et al. 2008: 501-502).

La compétition n'excuse pas tout. Afin de faire comprendre ce que cette conclusion a de choquant je rappellerai trois choses. Premièrement, le concept d'homoplasie a été conçu - il y a longtemps, en 1870 - par un morphologiste (Edwin Lankester). Deuxièmement, un siècle plus tard le premier algorithme de parcimonie fonctionnant sans contrainte sur la partition homologie-homoplasie fut élaboré par des morphologistes (Arnold Kluge et Steve Farris). Troisièmement, les approches probabilistes à la source de presque tous les résultats modernes de phylogénie moléculaire - notamment ceux de Springer et al. (2007) - n'indiquent jamais précisément quel est ce rapport homologie-homoplasie (autrement dit l'homoplasie minimale dans les données); au contraire elles sont fondées sur une modélisation du comportement des nucléotides, ce qui est une manière de résoudre le problème de la saturation que je qualifierais, prudemment, de contournée (voir Debruyne \& Tassy 2004). Quant à l'information exceptionnelle qui serait celle fournie par des insertions-délétions rares dans les séquences d'introns - rareté qui est d'ailleurs plus supposée que calculée -, elle est malheureusement nuancée à l'intérieur même des cercles moléculaires (rappelons l'exemple des Pegasoferae cité ci-dessus). Le couple homologie-homoplasie n'a pas fini de titiller les phylogénéticiens, toutes spécialités confondues.

Laissons la conclusion à Edgar A. Poe: «Savez-vous qu'il n'y a guère plus de huit ou neuf cents ans que les métaphysiciens ont consenti pour la première fois à délivrer le peuple de cette étrange idée : qu'il n'existait que deux routes praticables conduisant à la Vérité? Croyez cela, si vous le pouvez!» (Poe 2007: 14).

\section{CONCLUSION}

Je me souviens des premières discussions dans les années 1970 entre Robert Hoffstetter (alors directeur du laboratoire de paléontologie des vertébrés et de paléontologie humaine de l'Université Paris 6) et Louis de Bonis (alors maître-assistant) - auxquelles, tout jeune assistant, j'assistai plus que je n'y participai - sur l'intérêt à accorder aux travaux pionniers de phylogénie moléculaire menés par l'équipe de Morris Goodman à Detroit, USA. Robert Hoffstetter était enthousiaste, Louis de Bonis plus circonspect. Aujourd'hui, curieusement, on peut affirmer que rien n'a changé dans le grand écart caractérisant les attitudes extrêmes. Qu'il s'agisse de la phylogénie moléculaire à l'intérieur du genre Mammuthus ou bien celle des Mammalia dans leur ensemble, les résultats inattendus ou contradictoires de cette année 2008 ont de quoi amplifier le débat et susciter la recherche. Sauf si les arguments quittent le terrain de la discussion scientifique comme on vient de le voir à propos des Mammalia. Ignorer, en fonction de sa spécialité, les données issues du monde moléculaire ou bien du monde 
paléontologique, n'a guère de sens. Au contraire, tenir compte de la contradiction est plus riche sur le plan des recherches à venir; mais c'est peut-être plus délicat et frustrant en termes de trajectoire à tenir. Le poids sociologique des communautés scientifiques n’a jamais été aussi lourd. Par chance pour les paléontologues l'ADN de mammouth est de l'ADN ancien, il n'existerait donc pas sans paléontologie: on peut en tirer, selon le contexte, une sorte de fierté ou de consolation!

\section{Remerciements}

Merci à Stéphane Peigné (MNHN) et Gildas Merceron (Université Claude Bernard, Lyon) qui m’ont invité à participer à ce volume jubilaire en l'honneur de Louis de Bonis qui fut mon mentor à l'époque du DEA de Paléontologie des Vertébrés et de Paléontologie humaine à l'Université Paris 6 en 1971-1972, le moment clé où la vocation enfantine de paléontologue, quelque peu fantasmatique, se transforme en choix professionnel dûment réfléchi. Merci également à Philippe Taquet (MNHN) qui a attiré mon attention sur la précieuse correspondance entre Marcellin Boule et Henry Fairfield Osborn conservée au Muséum. J'ai bénéficié des conseils et suggestions de Régis Debruyne (McMaster University, Canada) qui a lu une première version de ce manuscrit. Cet article a été amélioré grâce aux critiques constructives de U. Göhlich, de A. Ohler et d'un rapporteur anonyme. Les idiosyncrasies qui caractérisent cet article restent les miennes, par définition.

\section{RÉFÉRENCES}

Asher R. J., Geisler J. H. \& SAnchez-Villagra M. R. 2008. - Morphology, paleontology, and placental mammal phylogeny. Systematic Biology 57: 311-317.

Bajgusheva V. S. \& Titov V. V. 2001. - [The review of the fossil elephant's findings from the coast of Azov sea], in Rozanov A. Y. (ed.), [Mammoth and its Environment: 200 Years of Investigations]. GEOS, Moscou: 81-71 (en russe, résumé anglais).

Barnes I., Shapiro B., Lister A., Kuznetsova T., Sher A., Guthrie D. \& Thomas M. G. 2007. Genetic structure and extinction of the woolly mam- moth, Mammuthus primigenius. Current Biology 17: 1072-1075.

BARriel V., ThueT E. \& TASSY P. 1999. - Molecular phylogeny of Elephantidae. Extreme divergence of the extant forest African elephant. Comptes Rendus de l'Académie des Sciences, Paris, Sciences de la Vie/Life Sciences 322: 447-454.

BOCHERENS H. 2003. - Isotopic biogeochemistry and the paleoecology of the mammoth steppe fauna, in Reumer J. W. F., De Vos J. \& Mol D. (eds), Advances in mammoth research. Proceedings of the Second International Mammoth Conference, Rotterdam, May 16-20 1999, Deinsea 9: 57-76.

BONDE N. 2001. — L'espèce et la dimension du temps, in TASSY P. \& DE RICQLÈS A. (eds), Systématique et paléontologie. Biosystema 19. Société Française de Systématique, Paris: 29-62.

Breyne J. P. 1741. - A letter from John Phil. Breyne M.D. F.R.S. to sir Hans Sloane, Bart. Pres. R.S. with observations, and a description of some mammoth's bones dug up in Siberia, proving them to have belonged to elephants. Philosophical Transactions of the Royal Society London 40 (for the years 1737-1738): 124-138.

Capelli C., MacPhee R. D. E., Roca A. L., Brisighelli F., Georgiadis N., O’Brien J. O. \& Greenwood A. D. 2006. - A nuclear DNA phylogeny of the woolly mammoth (Mammuthus primigenius). Molecular Phylogeny and Evolution 40: 620-627.

Churcher C. S. 1980. - Did the North American mammoths migrate? Canadian Journal of Anthropology 1: 103-105.

Cox P. G. 2006. - Character evolution in the orbital region of the Afrotheria. Journal of Zoology 269: 514-526.

Debruyne R., Barriel V. \& TAssy P. 2003. - Mitochondrial cytochrome $b$ of the Lyakhov mammoth (Proboscidea, Mammalia): new data and phylogenetic analyses of Elephantidae. Molecular Phylogeny and Evolution 26: 421-434.

Debruyne R., Chu G., King C. E., Bos K., Kuch M., Schwarz C., SzPaK P., Gröcke D. R., Matheus P., Zazula G., Guthrie D., Froese D., Buigues B., Marliave C. De, Flemming C., Poinar D., Fisher D., Southon J., Tikhonov A. N., MacPhee R. D. E. \& Poinar H. N. 2008. - Out of America: Ancient DNA evidence for a New World origin of Late Quaternary woolly mammoths. Current Biology 18: $1320-1326$.

Debruyne R., Schwarz C. \& Poinar H. 2008. Comment on "Whole-genome shotgun sequencing of mitochondria from ancient hair shafts". Science 322: 857.

DebruYne R. \& TASSY P. 2004. - Vers une phylogénétique non systématique? in CiBOIS A., BourgoIN T. \& SilvaIn J.-F. (eds), Avenir et pertinence des méthodes 
d'analyse en phylogénie moléculaire. Biosystema 22. Société française de Systématique, Paris: 25-34.

Derenko M., Malyartchuk B. \& Shields C. F. G. 1997. - Mitochondrial cytochrome $b$ sequence from a 33,000 year-old woolly mammoth (Mammuthus primigenius). Ancient Biomolecules 1: 149-153.

Dubrovo I. A. 1971. — Order Proboscidea, in NiKoforova K. V., Belyaeva E. I., Vangengeim E. A., Konstantinova N. A. \& Negadaev-NikOnov (eds), Pleistocene of Tiraspol. Académie des Sciences de Moldavie SSR, Kishinev: 92-108.

DudLEY J. P. 1996. — Mammoths, gomphotheres, and the Great American Faunal Interchange, in SHOSHANI J. \& TAssy P. (eds), The Proboscidea. Evolution and Palaeoecology of Elephants and their Relatives. Oxford University Press, Oxford: 289-295.

ELDREDGE N. 1971. - The allopatric model and phylogeny in Paleozoic invertebrates. Evolution 25: 156167.

Eldredge N. 1986. - Time Frames. Heinemann, Londres, $240 \mathrm{p}$.

Eldredge N. \& Gould S. J. 1972. - Punctuated equilibria: an alternative to phyletic gradualism, in Schopf T. J. M. (ed.), Models in Paleobiology. Freeman, Cooper \& Co, San Francisco: 82-115.

FABRE P.-H. 2008. - Inférence des processus de diversification au sein des Mammiferes: lintérêt des méthodes phylogénétiques macroévolutives appliqués aux Rongeurs et Primates. Thèse de Doctorat de l'Université de Montpellier II, France, 302 p.

FORONOVA I. V. 2001. - Quaternary Mammals of the South-East of Western Siberia (Kuznetsk Basin) Phylogeny, Biostratigraphy, and Paleoecology. Publishing House of SB Ras, Branch "Geo", Novosibirsk, 187 p.

GarutT V. \& TikHonov A. 2001. - [Origin and systematic of family Elephantidae Gray, 1821], in RozANOV A. Y. (ed.), [Mammoth and its Environment: 200 Years of Investigations]. GEOS, Moscou: 47-70 (en russe, résumé anglais).

Gatesy J., Milinkovitch M., Waddell V. \& StanHOPE M. 1999. — Stability of cladistic relationships between Cetacea and higher-level artiodactyl taxa. Systematic Biology 48: 6-20.

Gheerbrant E., Sudre J., Tassy P., Amaghzaz M., Bouya B. \& IAROChĖNE M. 2005. - Nouvelles données sur Phosphatherium escuilliei (Mammalia, Proboscidea) de l'Éocène inférieur du Maroc, apports à la phylogénie des Proboscidea et des ongulés lophodontes. Geodiversitas 27 (2): 229-333.

Gilbert M. T. P., Drautz D. I., Lesk A. M., Ho S. Y. W., Qi J., Ratan A., Hsu C.-H., Sher A., Dalén L., GÖtherström A., Tomsho L. P., RenduliC S., Packard M., Campos P. F., Kuznetsova T. V., Shidlovskiy F., Tikhonov A., Willerslev E., IACUMin P., Buigues B., ERICSON P. G. P., GERMONPRÉ M., Kosintsev P., Nikolaev V., NowaK-Kemp M.,
Knight J. R., Irzyk G. P., Perbost C. S., Fredrikson K. M., Harkins T. T., Sheridan S., Miller W. \& SCHUSTER S. C. 2008. - Intraspecific phylogenetic analysis of Siberian woolly mammoths using complete mitochondrial genomes, Proceedings of the National Academy of Sciences USA 105: 8327-8332.

Gilbert M. T. P., TOMSho L. P., Rendulic S., Packard M., Drautz D. I., Sher A., Tikhonov A., Dalén L., Kuznetsova T., Kosintsev P., Campos P. F., Higham T., Collins M. J., Wilson A. S., Shidlovskiy F., Buigues B., Ericson P. G. P., Germonpré M., GÖtherström A., IACUmin P., NikOlaev V., NowaKKemp M., Willerslev E., Knight J. R., IrZyK G. P., Perbost C. S., Fredrikson K. M., Harkins T. T., SHERIDAN S., Miller W. \& SCHUSTER S. C. 2007. Whole-genome shotgun sequencing of mitochondria from ancient hair shafts. Science 317: 1297-1930.

Gould S. J. \& Eldredge N. 1993. - Punctuated equilibrium comes of age. Nature 366: 223-227.

Greenwood A. D., CAPelli C., Possnert G. \& PÄÄBO S. 1999. - Nuclear DNA sequences from the Late Pleistocene megafauna. Molecular Biology and Evolution 16: 1466-1473.

Guérin C. \& Patou-Mathis M. 1996. — Les grands mammiferes plio-pléistocènes d'Europe. Masson, Paris, $291 \mathrm{p}$.

GUTHRIE R. D. 2004. — Radiocarbon evidence of midHolocene mammoths stranded on an Alaskan Bering Sea island. Nature 429: 746-749.

GuTHRIE R. D. 2006. - New carbon dates link climatic change with human colonization and Pleistocene extinctions. Nature 441: 207-209.

Hennig W. 1966. - Phylogenetic Systematics. The Illinois University Press, Urbana, 263 p.

Krause J., Dear P. H., Pollack J. L., Slatkin M., Spriggs H., BARNes L., Lister A. M., Ebersberger I. PÄÄBo S. \& Hofreiter M. 2006. - Multiplex amplification of the mammoth mitochondrial genome and the evolution of Elephantidae. Nature 439: 724-727.

KuZMin Y. V. \& Orlova L. A. 2004. — Radiocarbonchronology and environment of woolly mammoth (Mammuthus primigenius Blum.) in northern Asia: results and perspectives. Earth-Science Reviews 68: 133-169.

LABE B. \& Guérin C. 2005. - Réhabilitation de Mammuthus intermedius (Jourdan 1861), un mammouth (Mammalia, Elephantidae) du Pléistocène moyen récent d'Europe. Comptes Rendus Palevol 4: 235-242.

LisTer A. M. 1996. - Evolution and taxonomy of Eurasian mammoths, in SHOSHANI J. \& TASSY P. (eds), The Proboscidea. Evolution and Palaeoecology of Elephants and their Relatives. Oxford University Press, Oxford: 203-213.

LisTer A. M. 2001. — "Gradual" evolution and molar 
scaling in the evolution of the mammoth, in CAVARretTA G., Gioia P., Mussi M. \& Palombo M. R. (eds), La terra degli Elefanti/ The world of Elephants, Consiglio Nazionale delle Ricerche, Roma, 648-651.

LisTER A. M. \& JOYSEY K. A. 1992. — Scaling effects in elephant dental evolution - the example of Eurasian Mammuthus, in SMITH P. \& TCHERnOV E. (eds), Structure, Function and Evolution of Teeth. Freund, Tel-Aviv: 185-213.

Lister A. M. \& Sher A. 2001. - The origin and evolution of the woolly mammoth. Science 294: 1094-1097.

Lister A. M., Sher A. V., van Essen H. \& Wei G. 2005. - The pattern and process of mammoth evolution in Eurasia. Quaternary International 126128: 49-64.

Liv F-G. R. \& Miүaмото M. M. 1999. — Phylogenetic assessment of molecular and morphological data for eutherian mammals. Systematic Biology 48: 54-64.

Maglio V. 1973. - Origin and evolution of the Elephantidae. Transactions of the American Philosophical Society 63: 1-149.

Markov G. N. \& Spassov N. 2003. — Primitive mammoths from Northeast Bulgaria in the context of the earliest mammoth migrations in Europe, in PETRULESCU A. \& STIUCA E. (eds), Advances in Vertebrate Paleontology. "Hen to Panta", Bucarest: 53-58.

Matthee C. A., Eick G., Willows-Munro S., MontGELARD C., PARdini A. T. \& Robinson T. J. 2007. Indel evolution of mammalian introns and the utility of non-coding nuclear markers in eutherian phylogenetics. Molecular Phylogeny and Evolution 42: 827-837.

Miller W., Drautz D. I., Ratan A., Pusey B., Qi J., Lesk A. M., Tomsho L. P., Packard M. D., ZhaO F., Sher A., Tikhonov A., Raney B., Patterson N., Lindblad-Toh K., Lander E. S., Knight J. R., IrZyK G. P., Fredrikson K. M., Harkins T. T., Sheridan S., Pringle T. \& SChuster S. C. $2008 .-$ Sequencing the nuclear genome of the extinct woolly mammoth. Nature 456: 387-390.

NichOlLs H. 2008. — Let's make a mammoth. Nature 456: 310-314.

Nishihara H., HasegaWA M. \& OKADA N. 2006. Pegasoferae, an unexpected mammalian clade revealed by tracking ancient retroposon insertions. Proceedings of the National Academy of Sciences USA 103: 9929-9934.

Noro M., Masuda R., Dubrovo I., Yoshida M. C. \& KATO M. 1998. - Molecular phylogenetic inference of the woolly mammoth Mammuthus primigenius, based on complete sequences of mitochondrial cytochrome $b$ and $12 S$ ribosomal RNA. Journal of Molecular Evolution 46: 314-326.

Osborn H. F. 1933a. - Biological inductions from the evolution of the Proboscidea. Proceedings of the National Academy of Sciences USA 19: 159-163.
Osborn H. F. 1933b. - Aristogenesis, the observed order of biomechanical evolution. Proceedings of the National Academy of Sciences USA 19: 699-703.

Osborn H. F. 1942. - Proboscidea. Volume 2, Stegodontoidea Elephantoidea. The American Museum Press, New York: xxvii + 805-1676.

Ozawa T., Hayashi S. \& Mickelson V. M. 1997. — Phylogenetic position of mammoth and Steller's sea cow within Tethytheria demonstrated by mitochondrial DNA sequence. Journal of Molecular Evolution 44: 406-413.

Penny D., Hasegawa M., Waddell P. J. \& Hendy M. D. 1999. - Mammalian evolution: timing and implications from using the LogDeterminant Transform for proteins of differing amino acid compositions. Systematic Biology 48: 76-93.

Poe E. A. 2007. - Eurêka. (Trad. Charles Baudelaire; postface de Willam T. Vollmann). Tristram, Auch, $152 \mathrm{p}$.

Poinar H. K., Schwarz C., Qi J., Shapiro B., MacPhee R. D. E., Buigues B., TikHonov A., Huson D., Tomsho L. P., Auch A., Rampp M., Miller W. \& SCHUSTER S. C. 2006. - Metagenomics to paleogenomics: large-scale sequencing of mammoth DNA. Science 311: 392-394 (publié en ligne le 22/12/2005 sur Sciencexpress).

RINCON P. 2008. - "Rare" mammoth skull discovered. $B B C$ News. En ligne: http://news.bbc.co.uk/2/hi/ science/nature/7592317.stm

Rogaev E. I., Moliaka Y. K., Malyarchuk B. A., KONDRAShOV F. A., DerenKo M. V., ChUMAKOV I. \& GRIGORENKO A. P. 2006. — Complete mitochondrial genome and phylogeny of Pleistocene mammoth Mammuthus primigenius. PLoS Biology 4: e73.

Rose K. D. \& Archibald J. D. (eds) 2005. - The Rise of Placental Mammals. The Johns Hopkins University Press, Baltimore \& London, xi +259 p.

Savage R. J. G., Domning D. P. \& Thewissen J. G. M. 1994. - Fossil Sirenia of the West Atlantic and Caribbean region. V. The most primitive known sirenian, Prorastomus sirenoides Owen, 1855. Journal of Vertebrate Paleontology 14: 427-449.

Schreve D. C., KeEn D. H., Limondin-Lozouet N., Auguste P., Santisteban J. I., Ubilla M., Matoshio A., Bridgland D. R. \& Westaway R. 2007. - Progress in faunal correlation of Late Cenozoic fluvial sequence 2000-4: the report of the IGCP 449 biostratigraphy group. Quaternary Science Reviews 26 (22-24): 2970-2995.

SHOSHANI J. \& TASSY P. 2005. - Advances in proboscidean taxonomy $\&$ classification, anatomy $\&$ physiology, and ecology \& behavior. Quaternary International 126-128: 5-20.

Springer M. S., Amrine H. M., Burk A. \& Stanhope M. J. 1999. - Additional support for Afrotheria and Paenungulata, the performance of mitochondrial ver- 
sus nuclear genes, and the impact of data partitions with heterogeneous base composition. Systematic Biology 48: 65-75.

Springer M. S., Burk-Herrick A., Meredith R., Eizirik E., O’Brien S. J. \& Murphy W. J. 2007. The adequacy of morphology for reconstructing the early history of placental mammals. Systematic Biology 56: 673-684.

Springer M. S., Meredith R. W., EIZIRIK E., TeEling E. \& MurPHY W. J. 2008. - Morphology and placental mammal phylogeny. Systematic Biology 57: 499-503.

Stuart A. J., Kosintsev P. A., Higham T. F. G. \& Lister A. M. 2004. - Pleistocene to Holocene extinction dynamics in giant deer and woolly mammoth. Nature 431: 684-689.

Tabuce R., Marivaux L., Adaci M., Bensalak M., Hartenberger J.-L., Mahboubi M., Mebrouk F., TAFFOREAU P. \& JAEGER J.-J. 2007. — Early Tertiary mammals from North Africa reinforce the molecular Afrotheria clade. Proceedings of the Royal Society, Biology $274: 1159-1166$.

TASSY P. 1981. - Le crâne de Moeritherium (Proboscidea, Mammalia) de l'Eocène Dor el Talha (Libye) et le problème de la classification phylogénétique du genre dans les Tethytheria McKenna, 1975. Bulletin du Muséum national d'Histoire naturelle, 4e Série, Section C, $3:$ 87-147.

TASSY P. 2003. - Elephantoidea from Lothagam, in LEAKEY M. G. \& HARRIS J. M. (eds), Lothagam. The Dawn of Humanity in Eastern Africa. Columbia University Press, New York: 331-358.

TASSY P. 2004a. - Peut-on faire revivre le mammouth? Éditions Le Pommier, Paris, $61 \mathrm{p}$.

TASSY P. 2004b. — La famille des mammouths. Dossier
Pour la Science 43: 6-13.

Thomas M. G., Hagelberg E., Jones H. B., Yang Z. \& LisTER A. M. 2000. - Molecular and morphological evidence on the phylogeny of the Elephantidae. Proceedings of the Royal Society, London 267: 2493-2500.

VARTANYAN S. L., GaruTt V. E. \& SHER A. V. 1993. Holocene dwarf mammoths from Wrangel Island in the Siberian Arctic. Nature 362: 337-340.

Waddell P. J., CaO Y., Hauf J. \& Hasegawa M. 1999a. - Using novel phylogenetic methods to evaluate mammalian mtDNA, including amino-acid invariant sites-LogDet plus site stripping, to detect internal conflicts in the data, with special reference to the positions of hedgehog, armadillo, and elephant. Systematic Biology 48: 31-53.

Waddell P. J., OKada N. \& Hasegawa M. 1999b. Towards resolving the interordinal relationships of placental mammals. Systematic Biology 48: 1-5.

Wei G., Taruno H., Jin C. \& Xie F. 2003. — The earliest specimens of the steppe mammoth, Mammuthus trogontherii, from the Early Pleistocene Nihewan Formation, North China. Earth Science 57: 289-298.

WeSTOLL T. S. 1956. - The nature of fossil species, in Sylvester Bradley P. C. (ed.), The Species Concept in Palaeontology. Systematic Association Publication 2, Londres: 53-62.

Wible J. R., Rougier G. W., Novacek M. J. \& Asher R. J. 2007. - Cretaceous eutherians and Laurasian origin for placental mammals near the K/T boundary. Nature 447: 1003-1006.

Yang H., Golenderg E. M. \& Shoshani J. 1996. — Phylogenetic resolution within the Elephantidae using fossil DNA sequence from the American mastodon (Mammut americanum) as an outgroup. Proceedings of the National Academy of Sciences USA 93: 1190-1194.

Soumis le 19 décembre 2008; accepté le 30 octobre 2009. 